\title{
A Highlight on Reasons for Tattoo Regrets and Removal
}

\author{
Fabián Pérez Rivera
}

Department of Plastic Surgery, Cirugía \& Láser Dr. Pérez Rivera Medical Center, Buenos Aires, Argentina
Received December 18, 2020

Accepted March 15, 2021

\footnotetext{
Correspondence

Fabián Pérez Rivera

Department of Plastic Surgery, Cirugía \& Láser Dr. Pérez Rivera Medical Center, Bulnes 1937

(Zip Code 1425), Buenos Aires, Argentina

Tel.: +54-91151140231

Fax: +54-1148252217

E-mail: fabiandperezrivera.com.ar

(C) Korean Society for Laser Medicine and Surgery

(c) This is an open access article distributed under the terms of the Creative Commons Attribution NonCommercial License (http://creativecommons.org/ licenses/by-nc/4.0) which permits unrestricted noncommercial use, distribution, and reproduction in any medium, provided the original work is properly cited.
}

\author{
Background and Objectives \\ As the popularity of tattooing has increased, the number of people \\ regretting their tattoos, and therefore seeking tattoo removal services \\ has also increased. This study aimed to highlight the reasons for tattoo \\ regrets. We explore herein, the reasons for seeking tattoo removal at a \\ specialized laser center in South America.
}

\section{Materials and Methods}

This is a retrospective study of over 757 surveys, including patients who sought tattoo removal services between August 2017 and February 2020. The surveys were conducted using questionnaires which were sent to the patients through email and WhatsApp messages.

\section{Results}

Sixty percent of the patients seeking tattoo erasing services were women $(\alpha=0.001)$, an average of 30 years, with a high educational level $(46.6 \%$ of them were university or tertiary). Most of the tattoos (89\%) had a professional origin, $60 \%$ were done with black color and $65 \%$ were in exposed areas. More than half of the patients (51\%) gave dissatisfaction as the main reason for erasing their tattoos. More than half of patients $(51.2 \%)$ regretted their tattoos between the same day of being tattooed and five years later.

\section{Conclusion}

In the present study, in South America, tattoo regrets were mostly common among educated (university or tertiary level) women in their 30s. The most preferred color for a tattoo was black. Exposed areas, especially the arms, were preferred more than the covered areas. Most of the participants regretted their tattoos within five years of being tattooed.

\section{Key words}

Laser tattoo removal; Tattoo removal; Tattooed regret; Tattooed regret profile; Tattooed population characteristics 


\section{INTRODUCTION}

Tattooing has been practically a ubiquitous practice whose purposes vary from culture to culture, place, and time. In the past, tattoos served as rites of passage, religious symbols, decorations for bravery or punishments, and marks of outcasts and slaves.' Similarly, today, the motivations for acquiring a tattoo vary, including change of appearance, assertiveness, self-esteem, and expression of uniqueness. ${ }^{2}$

The prevalence of tattoos in the USA is estimated to be $30 \%$ of the general population, and $40 \%$ of Americans aged $26-40 .^{3}$ Owing to the increased acceptance, tattoos' popularity has been growing. In 2005 Armstrong et al. found an annual increase of $55 \%$ since 1996 in Texas registered tattoo studio. ${ }^{4}$ In Europe, from 2003 to 2012 for the general population, and for the men and women, tattoos prevalence rose from $10 \%$ to approximately $15-25 \%$ among the youths. ${ }^{5}$

Over time, some people regret getting tattoos or a specific type of tattoo and wish to have them erased.

In 2018, DALIA, a German consultancy, carried out an internet survey in 18 countries (Argentina, Australia, Brazil, Canada, Germany, Denmark, Spain, Sweden, United States, England, Greece, Denmark, France, Israel, Mexico, Russia, Turkey, and South Africal to find out the popularity and perception of tattoos in the world. They received a total of 9054 responses, a reasonably significant number to make a valid conclusion. 38\% of the respondents had at least one tattoo. Italy had the highest number of respondents with tattoos, followed by Sweden and the United States, and Argentina was fourth. 28\% of the respondents with tattoos regretted at least one of their tattoos. ${ }^{6}$

The highest number of tattoos was three. Surprisingly, "you should have an odd number of tattoos because it brings luck," a famous saying by the legend of Otzi, a found mummy dating back to 3000 years B.C. who had 57 tattoos, was influential among the respondents. ${ }^{3}$

As indicated by the mounting evidence, as the number of tattooed people increases, people who regret their tattoos or seek to improve or modify them increase. Similarly, with the evolution of tattooing, the techniques for removing tattoos have progressed in parallel. Tattoo removal methods include surgical excision, salabrasion, chemical peel, caustic or infrared coagulation, dye laser, $\mathrm{CO}_{2}$ laser, and Q-switched laser. Among them, Qswitched laser has been the method of choice for the majority and the most commonly performed. ${ }^{7-9}$ More recently, the $\mathrm{Q}$-switched laser picosecond, an improved technology from the traditional Q-switched Lasers, is the most popular method of tattoo removal. 3.10 $^{-10}$

This study aims to highlight some of the reasons for tattoo regrets.

Herein, it evaluated the characteristics of patients seeking tattoo removal services at a South American specialized laser center.

\section{MATERIALS AND METHODS}

This is a retrospective study of over 757 surveys, including patients who consulted for tattoo removal between August 2017 and February 2020. The surveys were conducted using questionnaires which were sent to the patients through email and WhatsApp messages.

The questionnaires included the following questions:

1) Age

2) Age when the tattoo was done

3) Sex

4) Level of education

5) If the tattoo was of professional or amateur origin

6) Principal reason for erasing the tattoo

7) Body area where the tattoo was done

8) Tattoo color/colors

9) A history of laser treatment

\section{RESULTS}

The participants' age was between 15 to 69 years. The average was 30 years.

All the questionnaires were answered and sent to the medical laser center. Only 17 patients didn't indicate the time their tattoos were done. Sixty percent of patients (454 patients) seeking to erase their tattoos were women.

There was a high prevalence of tattoos among the young participants; at the same time, the number of participants who regretted their tattoos was also high in this group: 346 (45.7\%) were below 20 years when they were tattooed, and $81.3 \%$ were tattooed between their teen years and 30 years. Information on age distribution is presented in Table 1 and 2.

Half of the tattooed participants who wanted to erase had a high education level: 46.6\%, 353 patients had university or tertiary education level.

The majority of the tattoos had a professional origin: 89\%; and a high prevalence in women. Amateur tattoos were prevalent in women too (46 over a total of 83), except in the group below 20 years in which the number of males with amateur tattoos was higher than females (30:27). 
Table 1. Comparation between age tattoo regret and age tattoo were made

\begin{tabular}{|c|c|c|c|c|}
\hline & Total & Female & Male & \\
\hline $\mathrm{N}$ & 757 & $457(0.60)$ & $300(0.40)$ & $\alpha=0.001$ \\
\hline \multicolumn{5}{|c|}{ Age at time to repentance } \\
\hline Average & & $31(8.56)$ & $30.1(7.87)$ & $p>0.05$ \\
\hline Min & & 15 & 17 & \\
\hline Max & & 69 & 60 & \\
\hline $\mathrm{N}$ & 744 & 449 & 295 & \\
\hline \multicolumn{5}{|c|}{ Age at the time of tattooing } \\
\hline Average & & $24.0(8.87)$ & $22.8(7.36)$ & $p>0.05$ \\
\hline Min & & 11 & 12 & \\
\hline Max & & 63 & 53 & \\
\hline
\end{tabular}

Table 2. Age distribution at the time when the tattoo were made

\begin{tabular}{ccc}
\hline Age & Number of patients & Sex distribution \\
\hline 10 to 20 years & $346(45.7 \%)$ & $202 \mathrm{~F} / 144 \mathrm{M}$ \\
21 to 30 years & $270(35.6 \%)$ & $167 \mathrm{~F} / 103 \mathrm{M}$ \\
31 to 40 years & $89(11.7 \%)$ & $53 \mathrm{~F} / 36 \mathrm{M}$ \\
41 to 50 years & $27(3.5 \%)$ & $20 \mathrm{~F} / 7 \mathrm{M}$ \\
51 to 60 years & $7(0.9 \%)$ & $6 \mathrm{~F} / 1 \mathrm{M}$ \\
63 years & $1(0.13 \%)$ & $1 \mathrm{~F}$ \\
\hline
\end{tabular}

More than half of the patients (51\%) stated dissatisfaction as the main reason for erasing their tattoos. The second most common reason was a professional requirement (19.4\%), and the third most common reason was to change to significant others (14.9\%). Twenty-eight cases (3.7\%) only searched to fade colors, re-tattooed or eliminate some tattoo sections. Four participants were seeing to erase their tattoos due to an allergic reaction to the tattoo pigments. Information on the reasons for erasing tattoos is presented in Table 3.

The most frequent tattoo color was black (60\%). The second most frequent color was multicolor (more than three colors in the same tattoo) (19\%).

A majority of participants regretted their tattoos shortly after they were tattoed. Nine percent of them regretted their tattoos within the first year, and the majority (42.2\%) between 1 to 5 years after they were tattooed; Considering these two groups, our results indicated that more than half of the participants (51.2\%) regret tattoo between the same day and five after being tattooed.

Exposed areas larms, forearm, wrist, hands, leg, ankle, neck, and face) were the most preferred for tattoos (66\%), then the covered areas (thorax, back, abdomen, thighs, genital, and buttock).

Among the exposed areas, the most preferred was the arm (18\%); this includes the entire upper limb larm + forearm + wrist + hands). Similarly, a significant num-
Table 3. Main reasons for tattoo removal

\begin{tabular}{lc}
\hline Dissatisfied with the tattoo or how was make it & $387(51 \%)$ \\
Labor reasons & $147(19.4 \%)$ \\
Change significant other & $111(14.6 \%)$ \\
Boredom & $79(10.4 \%)$ \\
Reduce color for re-tattooed & $28(3.7 \%)$ \\
Allergic reaction to pigment & $5(0.6 \%)$ \\
\hline
\end{tabular}

Table 4. Tattooed body areas

\begin{tabular}{lrr}
\hline Entire upper limb & 391 & $51.6 \%$ \\
Arm & 134 & $17.7 \%$ \\
Forearm & 96 & $12.6 \%$ \\
Wrist & 68 & $9 \%$ \\
Hand & 63 & $8.3 \%$ \\
Back & 49 & $6.4 \%$ \\
Neck & 43 & $5.7 \%$ \\
Shoulder & 43 & $5.7 \%$ \\
Leg & 42 & $5.6 \%$ \\
Face & 35 & $4.65 \%$ \\
Fingers & 30 & $3.9 \%$ \\
Abdomen & 29 & $3.8 \%$ \\
Chest & 25 & $3.3 \%$ \\
Eyebrow & 17 & $2.2 \%$ \\
Waist & 11 & $1.5 \%$ \\
Others areas & 41 & $5.4 \%$ \\
\hline
\end{tabular}

ber of regrettable tattoos (33\%) were on the arms: 1 in 3 seeks to erase a tattoo in these areas. Information on the areas of regrettable tattoos is presented in Table 4.

Eighteen percent of the patients who sought tattoo removal services had previously undergone laser treatment in other centers or doctors' offices.

\section{DISCUSSION}

Despite increasing cases of tattoo removal, little has known regarding the characteristics and the reason tattoo regrets.

Traditionally, tattooing was more prevalent among older men, with its purposes social and behavior related. However, over the past 30 years, this trend has progressively changed. At the beginning of the 1990s, half of all tattoos were done by women across different social classes. ${ }^{1}$ In 2018 Dalia, a German consultancy found that $60 \%$ of individuals seeking to erase their tattoo were women, an average of 30 years. Half of them had a high educational level; $47 \%$ had university or tertiary education levels. ${ }^{6}$ Cosmetic tattoos are usually performed on women's faces (eyelids, eyebrows, and lips) for various reasons. They are an additional explanation of the increased prevalence of tattooing among women, across 
different age groups. ${ }^{11}$ However, this study included four males who performed cosmetic tattoos to simulate hair on their frontline forehead.

Sixty percent of patients (454 patients) seeking to erase their tattoos were women. If it takes as a value the last official census of the Argentine population (2010), out of a total $40,117,096$ population, $19,523,766$ (48.6\%) were men, and 20,593,330 were women (51.4\%). It makes relevant the higher percentage that we find of women who regret tattoos compared to men, $60: 40(\alpha=0.001)^{12}$

In the present study, the age range of individuals who sought regretted their tattoos is $15-69$ years. It is in agreement with Latreille et al., 2011; they concluded that anyone could regret their tattoos regardless of their life stage. ${ }^{13}$ The average age is 30 years. Notably, this life stage would coincide with a change of partner, job change, or enough time to get bored with one's tattoo. One of the possible reasons is the availability of more information on the progress in laser tattoo removal from social media and the Internet; the young population is more exposed to social media and the Internet in general than the older population.

Half of the participants (47\%) who sought tattoo removal or modification services had a high education level luniversity or tertiary levell. Parallel to these findings, most tattoos (89\%) had a professional origin that corresponds with higher education and economic levels.

In contrast with a majority of the references, in the present study, amateur tattoos were more common in women than in men ( 46 over a total of $83,55 \%$ ).

More than half of the participants (52\%) stated dissatisfaction as the main reason for erasing their tattoos: it was not what they had asked the tattoo artist, it was not as they expected it to be, the tattoo changed with time, tattoo artist mistakes (the most common mistake for a letter or sign), the tattoo no longer represents the same as in the past. The second most common reason (20\%) was professionally related, mainly those who want to enter some security or armed forces (police, army, gendarmerie, etc.).These forces do not allow tattoos in exposed areas such as the face, neck, arms, forearms, hands, legs, or feet. The third most common reason was a change of partner (14\%). They had tattooed names, initials, or figures representing their previous love relationship, and their current partner was uncomfortable with the tattoo's meaning.

It is noteworthy to mention that not all individuals seek to erase their tattoo: $5 \%$ only seek to fade colors, retattoo, or eliminate some small sections of their tattoos.

In the present study, four participants sought tattoo removal services due to an allergic reaction to the tattoo pigments. These allergic reactions occur because some red tones inks are made from mercury sulfide. The severity of the allergic reactions could get to an extent of interfering with individuals daily activities, the symptoms include, itching, pain, transudates, and scar due to scratching, which can transform to keloids. ${ }^{14,15}$

While the average age (30 years) for getting tattoos in the present study is similar to that of the references, the trend is different with the age when individuals regret their tattoos: unlike the studies done by Armstrong et al. in 1006 and 2006 where their study population took approximately 14 years and 10 years respectively to remove their tattoo, in this study, $53 \%$ of the participants regretted their tattoos within 1 to 5 years, and $10 \%$ of the participants regretted their tattoos within the first year after being tattooed.

Exposed areas larms, forearm, wrist, hands, neck, and face) were the more preferred tattooing areas (65.1\%) than the covered areas (thorax, back, abdomen, leg, thighs, feet, genital, breast, ankle, buttock). The arm $(17.7 \%)$ was the most frequently exposed tattooed area. More than half of the regrettable tattoos (51.6\%) were on one of the areas of the entire upper limb larm + forearm + wrist + hand + finger). This classification is subject to bias as the author's conclusion is based on individuals' clothing. More information is presented in Table 4.

Eighteen percent of the participants who sought tattoo removal services had previously undergone laser treatment in other centers or doctors' offices; reasons included dissatisfaction on improvement with different lasers, more information on tattoo advances from the Internet, or influence by friends and colleagues.

As the tattooed population increases worldwide, the tattoo regrets population increases. Based on our findings, a quarter of the world's population has at least one tattoo, and a quarter of all tattooed individuals regret their tattoos and seek to improve or erase them, at some point in their life.

Despite some limitations in this study, our findings provide insightful information to help individuals make informed decisions before getting a tattoo. In addition, our present study provides a foundation for further studies on reasons for tattoo regrets in other laser centers and other countries.

\section{CONCLUSION}

Based on the present study findings, in South America, tattoo regrets are mostly common among educated luni- 
versity or tertiary levell women in their 30s. The most preferred color was black, exposed areas, especially the arm, which were preferred more than the covered areas. The majority of the participants regretted their tattoos within five years of being tattooed. Social media and the Internet plays a big role as the main sources of information on laser tattoo technology.

\section{CONFLICT OF INTEREST}

The author declares that he has no conflict of interest.

\section{REFERENCES}

1. Armstrong ML, Roberts AE, Koch JR, Saunders JC, Owen DC, Anderson RR. Motivation for contemporary tattoo removal: a shift in identity. Arch Dermatol 2008;144:879-84.

2. Anderson R. Regarding tattoos: is that sunlight, or an oncoming train at the end of the tunnel? Arch Dermatol 2001;137:2102.

3. Hsu VM, Aldahan AS, Mlacker S, Shah W, Nouri K. The picosecond laser for tattoo removal. Lasers Med Sci 2016;31:17337.

4. Armstrong ML. Tattooing, body piercing, and permanent cosmetics: a historical and current view of state regulations, with continuing concerns. J Environ Health 2005;67:38-43, 54, 53.

5. Kluger N. Epidemiology of tattoos in industrialized countries. Curr Probl Dermatol 2015;48:6-20.

6. Armstrong M. DALIA research. Where tattoos are most popular [Internet]. Statista; 2018. [cited 2018 May 23]]. Available from: https://www.statista.com/chart/13942/where-tattoos- are-most-popular/.

7. Laumann AE, Derick AJ. Tattoos and body piercings in the United States: a national data set. J Am Acad Dermatol 2006;55:413-21.

8. Huxley C, Grogan S. Tattooing, piercing, healthy behaviours and health value. J Health Psychol 2005;10:831-41.

9. Armstrong ML, Roberts AE, Koch JR, Saunders JC, Owen DC. Investigating the removal of body piercings. Clin Nurs Res 2007;16:103-18.

10. Torbeck RL, Schilling L, Khorasani H, Dover JS, Arndt KA, Saedi N. Evolution of the picosecond laser: a review of literature. Dermatol Surg 2019;45:183-94.

11. Armstrong ML, Saunders JC, Roberts AE. Older women and cosmetic tattooing experiences. J Women Aging 2009;21:18697.

12. WebINDEC. Composition and Distribution: Population Census 2010. Buenos Aires: National Institute of Statistics and Census of Argentina; 2010.

13. Latreille J, Levy JL, Guinot C. Decorative tattoos and reasons for their removal: a prospective study in 151 adults living in South of France. J Eur Acad Dermatol Venereol 2011;25:181-7.

14. Serup J, Carlsen KH, Sepehri M. Tattoo complaints and complications: diagnosis and clinical spectrum. Curr Probl Dermatol 2015;48:48-60.

15. Kluger N. Cutaneous complications related to tattoos: 31 cases from Finland. Dermatology 2017;233:100-9.

How to cite this article: Pérez Rivera F. A highlight on reasons for tattoo regrets and removal. Med Lasers 2021;10:106-110. https://doi.org/10.25289/ML.2021.10.2.106 